\title{
Multiobjective TDMA Optimization for Neuron-based Molecular Communication
}

\author{
Junichi Suzuki \\ Dept. of Computer Science \\ University of Massachusetts, \\ Boston, USA \\ jxs@cs.umb.edu
}

\author{
Sasitharan Balasubramaniam \\ Telecommunication Software \\ Systems Group, Waterford \\ Institute of Technology, Ireland \\ sasib@tssg.org
}

\author{
Adriele Prina-Mello \\ School of Medicine \\ Trinity College Dublin, Ireland \\ prinamea@tcd.ie
}

\begin{abstract}
This paper proposes and evaluates Neuronal TDMA, a TDMAbased signaling protocol framework for molecular communication, which utilizes neurons as a primary component to build in-body sensor-actuator networks (IBSANs). Neuronal TDMA leverages an evolutionary multiobjective optimization algorithm (EMOA) that optimizes the signaling schedule for nanomachines in IBSANs. The proposed EMOA uses a population of solution candidates, each of which represents a particular signaling schedule, and evolves them via several operators such as selection, crossover, mutation and offspring size adjustment. The evolution process is performed to seek Pareto-optimal signaling schedules subject to given constraints. Simulation results verify that the proposed EMOA efficiently obtains quality solutions. It outperforms several conventional EMOAs.
\end{abstract}

\section{Categories and Subject Descriptors}

C.2.2 [Computer-Communication Networks]: Network Protocols; C.3 [Special-purpose and Application-based Systems]: Signal processing systems; I.2.8 [Artificial Intelligence]: Problem Solving, Control Methods, and SearchHeuristic methods

\section{General Terms}

Algorithms

\section{Keywords}

Molecular Communication, Neuronal networks, TDMA scheduling, Evolutionary multiobjective optimization algorithms

\section{INTRODUCTION}

Nanoscale communication is a new research paradigm that aims to provide communication capabilities between nanomachines. Nanomachines are the most basic functional unit in nanoscale systems. Their scale ranges from one to a few hundred nanometers. Each of them consists of biological materials (e.g., molecules) that perform very simple computation, sensing and/or actuation tasks [25].

The current research of nanoscale communication investigates molecular communication techniques as well as electromagnetic communication techniques. Molecular communication is inspired by the communication mechanisms that naturally occur among living cells. This bio-inspired communication paradigm utilizes molecules as a communication medium. Molecular communication has several advantages over electromagnetic communication such as inherent nanometer scale, biocompatibility and energy efficiency [7]. Given these advantages, one of major application domains of molecular communication is in-body or body area nanonetworks [1], where nanomachines are networked through molecular communication to perform sensing and actuation tasks in the body for biomedical purposes (e.g., vital information sensing and targeted drug release).

Molecular communication is often classified to short-range communication (nanometers to millimeters) and long-range communication (millimeters to meters) [7]. While a number of solutions have been proposed for short-range molecular communication (e.g., molecular motors [19], calcium signaling [23] and bacteria communication [16]), research efforts on long-range communication are relatively limited [7].

This paper focuses on long-range molecular communication that utilizes neurons as a primary component to build in-body sensor-actuator networks (IBSANs). A neuron-based IBSAN consists of a set of nanomachines (e.g., bio-sensors and bio-actuators) and a network of neurons that are artificially formed into a particular topology. The proposed IBSAN architecture allows nanomachines to interface (i.e., activate and deactivate) neurons in a non-invasive manner and communicate to other nanomachines through a chain of neurons with electric and chemical signals.

This paper proposes and evaluates a communication protocol framework, called Neuronal TDMA, which performs single-bit Time Division Multiple Access (TDMA) scheduling for neuron-based IBSANs. Neuronal TDMA allows nanomachines to multiplex and parallelize neuronal signal transmissions while avoiding signal interference to ensure that signals reach the destination. It makes decisions of signaling schedules (i.e., when to activate neurons to trigger signal transmissions) for nanomachines with an evolutionary multiobjective optimization algorithm (EMOA) that evolves a set of solution candidates (or individuals) via genetic operators. Each individual represents a particular TDMA schedule for nanomachines with respect to time. The proposed EMOA 


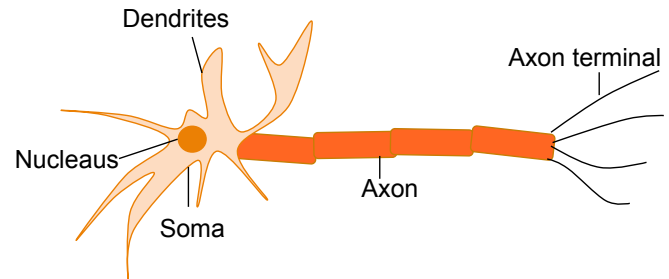

Figure 1: The structure of neurons

considers conflicting optimization objectives (e.g., signaling yield, signaling fairness among nanomachines and signaling delay $^{1}$ ) and seeks the optimal trade-offs among them subject to given constraints such as signaling delay.

Since there exists no single optimal solution (TDMA schedule) under conflicting objectives but rather a set of alternative solutions of equivalent quality, the proposed EMOA is designed to search Pareto-optimal solutions that are equally distributed in the objective space. Therefore, it can produce both extreme TDMA schedules (e.g., the one yielding high signaling rate and low signaling fairness) and balanced schedules (e.g., the one yielding intermediate signaling delay and intermediate signaling rate) at the same time. Given a set of heuristically-approximated Pareto-optimal TDMA schedules, the proposed EMOA allows the IBSAN designer to examine the trade-offs among them and make a wellinformed decision to choose one of them, as the best TDMA schedule, according to his/her preferences and priorities. For example, the IBSAN designer can examine how he/she can trade (or sacrifice) signaling yield for signaling fairness and determine a particular TDMA schedule that yields a desirable/comfortable balance of signaling yield and fairness.

Simulation results show that Neuronal TDMA efficiently obtains quality solutions with acceptable computational costs and allows nanomachines to perform neuronal signal transmissions while avoiding signal interference. It outperforms several well-known existing EMOAs.

\section{BACKGROUND}

This section describes the structural and behavioral properties of neurons. Neurons are fundamental components of the nervous system, which includes the brain and the spinal cord. They are electrically excitable cells that process and transmit information via electrical and chemical signaling.

The structure of a neuron is composed of a cell body (or soma), dendrites and an axon (Figure 1) [4]. The soma is the central part of a neuron. It can vary from 4 to 100 micrometers in diameter. Dendrites are thin structures that arise from the soma. They form a complex "dendrite tree" that extends the farthest branch a few hundred micrometers from the soma. Dendrites are where the majority of inputs to a neuron occur. An axon is a cellular extension that arises from the soma. It branches hundreds, or even thousands, of times before it terminates and travels through the body in bundles called nerves. Its length can be over one meter in the human nerve that arises from the spinal cord down to a toe.

Neurons self-organize to connect to each other and form

\footnotetext{
${ }^{1}$ For example, improving signaling yield can degrade signaling fairness. On the contrary, improving signaling delay can degrade signaling yield.
}

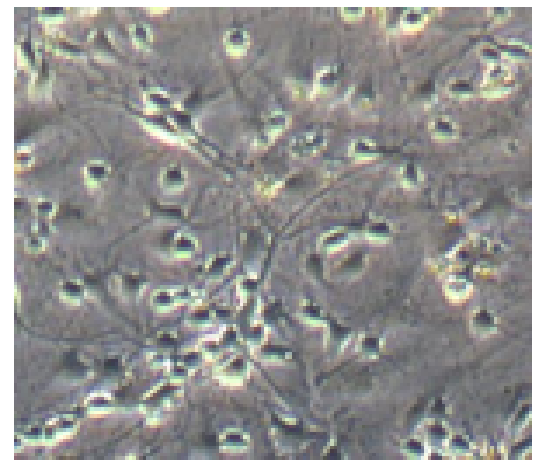

Figure 2: A Network of neurons. They were dissociated from 1-day old Ham-Wistar rats as described in [18]. Notice cell bodies, dendrites (smaller filaments) and axons (larger filaments) (magnification: $\times 20)$.

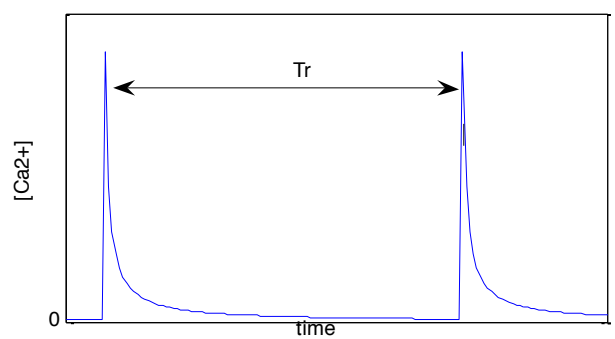

Figure 3: Intercellular $\mathrm{Ca}^{2+}$ concentration in a neuron. $\mathrm{Ca}^{2+}$ releases must be separated by at least the refractory time $T_{r}$.

networks [6]. Figure 2 shows a natural neuronal network that neurons have self-organized into. Neurons can communicate with others via synapses, each of which is a membraneto-membrane junction between two neurons. A synapse contains molecular machinery that allows a (presynaptic) neuron to transmit a chemical signal to another (postsynaptic) neuron. In general, signals are transmitted from the axon of a presynaptic neuron to a dendrite of a presynaptic neuron. An axon transmits an output signal to a postsynaptic neuron, and a dendrite receives an input signal from a presynaptic neuron. An axon makes a large number of synaptic contacts to neurons as it branches.

Neurons maintain voltage gradients across their membranes by means of voltage-gated ion channels, which are embedded in the presynaptic membrane to generate the differences between intracellular and extracellular concentration of ions (e.g., $\mathrm{Ca}^{2+}$ ) [24]. Changes in the cross-membrane ion concentration (i.e., voltage) can alter the function of ion channels. If the concentration (i.e., voltage) changes by a large enough amount (e.g., approximately $80 \mathrm{mV}$ in a giant squid), ion channels initiate a voltage-dependent process; they pump extracellular ions inward. Upon the increase in intracellular ion concentration, the presynaptic neuron releases a chemical called a neurotransmitter (e.g., acetylcholine $(\mathrm{ACh})$ ), which travels through the synapse from the presynaptic neuron's axon terminal to the postsynaptic neuron's dendrite. The neurotransmitter electrically excites the 
postsynaptic neuron, and the neuron generates an electrochemical pulse called an action potential. This signal travels rapidly along the neuron's axon and activates synaptic connections (i.e., opens ion channels) when it arrives at the axon's terminals. This way, an action potential triggers cascading neuron-to-neuron communication.

Figure 3 shows how intercellular $\mathrm{Ca}^{2+}$ concentration changes in a neuron. When the concentration peaks, the neuron releases a neurotransmitter to trigger an action potential. Once this process is activated, the neuron goes into a refractory period ( $T_{r}$ in Figure 3 ), which is the time required for the neuron to replenish its internal $\mathrm{Ca}^{2+}$ store. During $T_{r}$, it cannot process any other incoming signals (action potentials) from other neurons. The refractory period is approximately two milliseconds in a giant squid.

\section{NEURON-BASED IBSANS}

This section overviews the architecture and potential applications of in-body sensor-actuator networks (IBSANs) built with neuronal networks. This section also describes several assumptions and approaches to form neuronal networks into particular topology shapes and interface nanomachines to neurons for neuronal signaling.

\subsection{The Architecture of Neuron-based IBSANs}

This paper focuses on neuron-based molecular communication for IBSANs. Compared with other forms of molecular communication, neuron-based communication has three advantages: long distance coverage, high speed signaling (up to $90 \mathrm{~m} / \mathrm{s} \mathrm{[11])} \mathrm{and} \mathrm{low} \mathrm{attenuation} \mathrm{in} \mathrm{signaling} \mathrm{[7].}$

This paper focuses solely on artificial neuronal networks, each of which is a network of natural neurons that are artificially grown and formed into particular topology patterns. Figure 4 illustrates two example IBSANs built with artificial neuronal networks. Each IBSAN contains an artificial neuronal network and several nanomachines such as sensors and sinks. Sensors use neuronal signaling to transmit sensing information to sinks. Sinks might work as transducers or actuators. In Figure 4, IBSANs are placed in the neural systems of the brain and along the spinal cord. As potential applications, prosthetic devices and medical rehabilitation devices could leverage neuron-based IBSANs to better perform sensing, transducing and actuation tasks in the environment.

This paper assumes that neuronal networks and nanomachines (e.g., sensors and sinks) are designed loosely-coupled and developed as independent units because nanomachines are intended to interact with neuronal networks in a noninvasive manner. This means that it is not required to insert carbon nanotubes into neurons so that nanomachines can trigger signaling. In the proposed IBSAN architecture, each sensor releases chemical agents so that a nearby neuron activates trans-membrane calcium signaling that in turn induces an action potential signal toward a sink along a neuronal network. Sensors are assumed to possess embedded nozzles to release chemical agents.

\subsection{Artificial Neuronal Networks}

A number of different approaches have been developed to grow neurons on substrates. For example, in [2], Nguyen$\mathrm{Vu}$ et al demonstrated the use of vertically aligned carbon nanofibers as an interface to neuronal networks. The authors were able to show that the carbon nano fibers can be

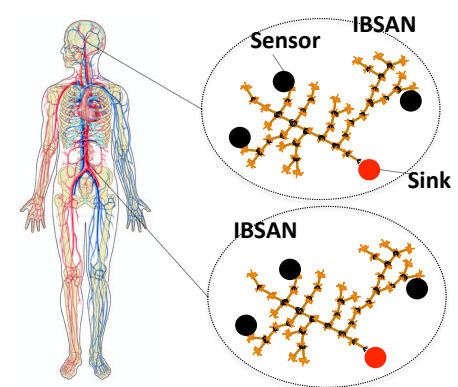

Figure 4: Example Neuron-based IBSANs

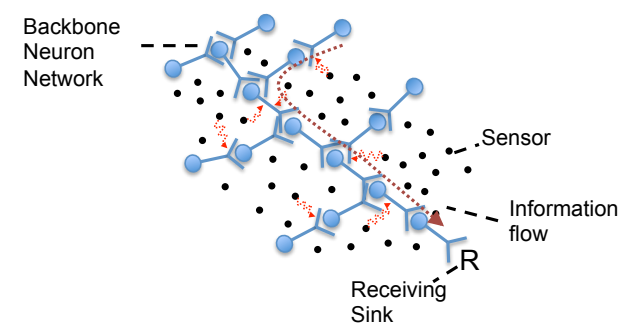

Figure 5: An Example of Neuronal Signaling

interfaced between the substrates and neuron cells, providing sufficient support to allow the neurons to comfortably grown and connect with other neurons. However, the challenge with our proposed approach not only lies on a assuming a compatible substrates to grow the neurons, but also to show that the neurons can be grown to specific topologies. This is important in our scheduling algorithm that will be discussed later, where we assume that the topology is known before hand, as well as the positions of the nano sensors, to optimally design the scheduling process of the nano sensors.

A number of works have focused on designing topologicallyspecific neuronal networks, many of which are applicable to our design. For example, in [20], Morin et al. developed a three-dimensional microfluidic system in polydimethylsiloxane (PDMS) were fabricated to allow neurons to be grown to specific patterns of topologies, where the fabricated PDMS were compatible with commercially available planar microelectrode arrays (pMEAs). Patterns include square grid chambers, where neurons were able to grow, and after a certain period, connection between the chambers were possible through axon growth from neurons in each chamber. Another work by Wyart [26] developed a similar solution for growing topology specific neuronal networks. The approach is based on polylysine patterns that confine the adhesion of cellular bodies to prescribed spots and the neuritic growth to specific thin lines. However, the work of S. B. Jun [13] fits our proposed approach very well, where the authors developed a solution of also using polylysine, but to grow the neurons in very low density. The structure is built with diameters of $20 \mu \mathrm{m}$ to allow cell soma to attach in specific locations. Our approach, that will be described in subsequent sections, is focused on the ability to invoke signaling on specific neurons for low density neuron networks.

\subsection{Neuronal Signaling}

As described in Section 3.1, the proposed IBSAN architecture employs a non-invasive process to trigger neuronal 
signaling. It assumes to use a non-invasive signaling process that the authors of this paper have developed in [2]. The prior work [2] carried out a series of wet lab experiments with cortical neuronal cultures plated on customized microelectrode arrays. Experimental results show that a chemical agent, acetycholine ( $\mathrm{ACh})$, can activate calcium signaling, which excites neurons and induces action potential signals. The results also show that calcium signaling can be suppressed by another chemical agent, mecamylamine. Therefore, the proposed IBSAN architecture assumes that each sensor possesses two nozzles that release ACh and mecamylamine to switch on and off neuronal signaling (Figure 5).

\section{NEURONAL TDMA}

If sensors emit ACh randomly to initiate calcium signaling, they may transmit signals on the same neurons at the same time. This leads to a large number of interference (or collisions) in the neuronal network, which in turn leads to corruption of transmitted information at the sink. As discussed above, neurons possess the refectory period in which no signals can be transmitted. Thus, Neuronal TDMA is intended to eliminate signaling interference through a chain of neurons toward the sink by scheduling which sensors activate which neurons with respect to time.

Neuronal TDMA is a single-bit TDMA protocol that periodically assigns a time slot dedicated to each sensor. Sensors activate neurons, one after the other, each using its own time slot. This allows multiple sensors to transmit signals to the sink through the shared neuronal network without interference. Each sensor transmits a single signal (a single bit) within a single time slot.

Figure 6 shows a simple example IBSAN that contains four nanomachines (three sensors and a sink) and a network of five neurons. Figure 7 depicts an example TDMA schedule for those sensors to activate neurons. The scheduling cycle period lasts 6 time slots $\left(T_{s}=6\right)$. The sensor $s_{1}$ activates the neuron $n_{4}$ to initiate signaling in the first time slot $T_{1}$. The signal travels through $n_{5}$ in the next time slot $T_{2}$ to reach the sink. The sensor $s_{2}$ transmits a signal on $n_{3}$ in $T_{2}$. During $T_{2}$, two signals travel in the neuronal network in parallel. The duration of each time slot must be equal to, or longer than, the refractory period $T_{r}$ (Figure 3 ).

The scheduling problem in Neuronal TDMA is defined as an optimization problem where a neuron-based IBSAN contains $M$ sensors, $S=\left\{s_{1}, s_{2}, \ldots, s_{i}, \ldots, s_{M}\right\}$, and $N$ neurons, $N=\left\{n_{1}, n_{2}, \ldots, n_{j}, \ldots, n_{N}\right\}$. Each sensor transmits at least one signals to the sink during the scheduling cycle $T_{s}$. $E^{s_{i}}=\left\{E_{1}^{s_{i}}, E_{2}^{s_{i}}, \ldots E_{k}^{s_{i}}, \ldots, E_{\mid E^{s_{i} \mid}}^{s_{i}}\right\}$ denotes the signals that a sensor $s_{i}$ transmits to the sink. $\left|E^{s_{i}}\right|$ is the total number of signals that $s_{i}$ transmits during the scheduling cycle $T_{s}$.

\subsection{Optimization Objectives and Constraints}

Neuronal TDMA considers three optimization objectives: (1) signaling yield, (2) signaling fairness among sensors and (3) signaling delay.

Signaling yield $(Y)$ is computed as follows. It is to be maximized.

$$
f_{Y}=\sum_{i=1}^{M}\left|E^{s_{i}}\right|
$$

This objective indicates the total number of signals that

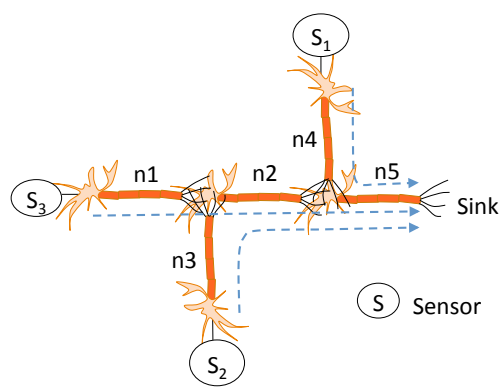

Figure 6: An Example IBSAN

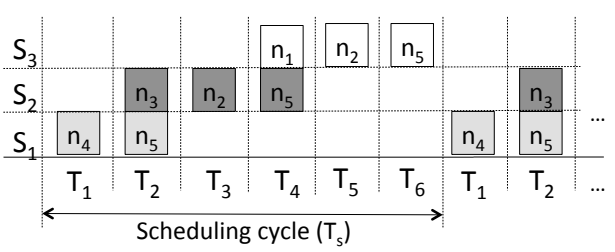

Figure 7: An Example TDMA Schedule

the sink receives from all $M$ sensors during the scheduling cycle $T_{s}$.

The second objective, signaling fairness $(F)$, is computed as follows. It is to be maximized.

$$
f_{F}=\sum_{l=1}^{M} \sum_{m=1}^{M} \sum_{k=1}^{\left|E^{s_{l}}\right|} \frac{1}{\left|t_{d}^{k\left(s_{l}\right)}-t_{d}^{k\left(s_{m}\right)}\right|}, \quad l \neq m
$$

$t_{d}^{k\left(s_{l}\right)}$ denotes the departure time of the $k$-th signal that $s_{l}$ transmits to the sink. This objective encourages sensors to equally access the shared neuronal network for signaling in order to avoid a situation where a limited number of sensors dominate the network. Higher fairness means that sensors access the neuronal network more equally.

The third objective, signaling delay $(D)$, is computed as follows. It is to be minimized.

$$
f_{D}=\max _{s_{i} \in S} t_{a}^{\left|E^{s_{i}}\right|\left(\left(s_{i}\right)\right.}
$$

$t_{a}^{\left|E^{s_{i}}\right|\left(\left(s_{i}\right)\right.}$ denotes the arrival time at which the sink receives the last (the $\left|E^{s_{i}}\right|$-th) signal that $s_{i}$ transmits. $f_{D}$ indicates how soon the sink receives all signals from all $M$ sensors. $f_{D}$ determines the scheduling cycle period $T_{s}$ $\left(T_{s}=f_{D}\right)$.

Neuronal TDMA considers three constraints in its optimization process. The first constraint enforces that at most one signal can pass through each neuron in a single time slot. The second constraint enforces each sensor transmit at least one signal to the sinks $\left(\left|E^{s_{i}}\right| \geq 1 \forall i=1,2, \ldots, M\right)$. The third constraint $\left(C_{D}\right)$ is the upper limit for $f_{D}$ : $f_{D} \leq C_{D}$. The delay constraint violation $\left(g_{D}\right)$ is computed as follows where $I=1$ if $f_{D}>C_{D}$ and $I=0$ otherwise.

$$
g_{D}=I \times\left(f_{D}-C_{D}\right)
$$

\subsection{Individual Representation}

In Neuronal TDMA, each individual represents a particular TDMA schedule for $M$ sensors. Figure 8 shows the 
structure of an individual. In this example, the first sensor, $s_{1}$, activates the first neuron $n_{1}$ for signaling. The signal travels through two neurons, $n_{2}$ and $n_{3}$, in the second and third time slots $t_{2}$ and $t_{3}$, respectively.

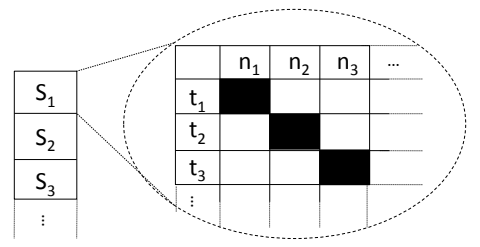

Figure 8: Individual Representation

\subsection{EMOA in Neuronal TDMA}

Figure 9 shows the algorithmic structure of the proposed EMOA in Neuronal TDMA. In the first generation $(t=0)$, $\mu$ individuals are randomly generated as the initial generation $P^{0}$. This process makes sure that generated individuals never violate constraints except the delay constraint $C_{D}$. In each generation $(t)$, a pair of individuals, called parents $\left(p_{1}\right.$ and $p_{2}$ ), are chosen from the current population $P^{g}$ using the binary tournament operator (BTounament()) [8]. A binary tournament randomly takes two individuals from $P^{t}$, compares them based on their fitness values, and chooses a superior one (i.e., the one whose fitness is higher) as a parent.

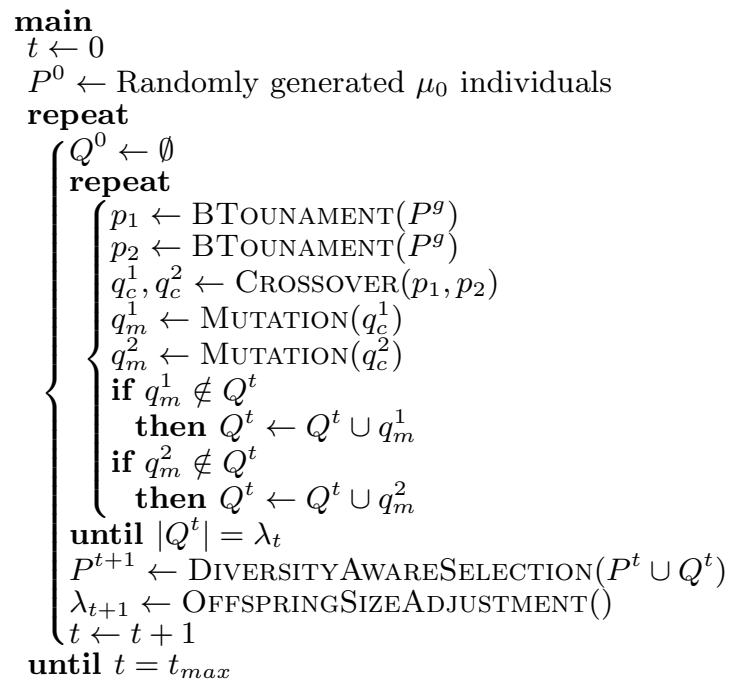

Figure 9: Algorithmic Structure of the Proposed EMOA in Neuronal TDMA

The notion of fitness is defined with constrained dominance relationships among individuals. The relationships rank individuals based on the objective values and delay constraint violation that they yield. An individual $i$ is said to constrained-dominate an individual $j$ if:

- $i$ does not violate the signaling delay constraint $(g D(i)=$ 0; c.f. Equation 4) but $j$ does $(g D(j)>0)$,

- both $i$ and $j$ do not violate the delay constraint, and $i$ dominates $j$ with respect to objectives, or
- both $i$ and $j$ violate the delay constraint, and the constraint violation of $i$ is less than $j$ 's $(g D(i)<g D(j))$.

Given the notion of dominance [5], $i$ is said to dominate $j$ (denoted by $i \succ j$ ) with respect to objectives if:

- $f_{k}(i) \leq f_{k}(j)$ for all $k=1,2, \ldots, m$, and

- $f_{k}(i)<f_{k}(j)$ for at least one $k \in 1,2, \ldots m$

$f_{k}(i)$ denotes the objective value that $i$ yields in the $k$-th objective. For $f_{Y}$ and $f_{F}$, their inverses are used here for an individual-to-individual comparison purpose because the two objectives are to be maximized.

Fitness is calculated for each individual $(i)$ as follows.

$$
\text { Fitness }(i)=\mu-d_{i}
$$

$\mu$ denotes the population size, and $d_{i}$ denotes the number of individuals that constrained-dominate $i$. Fitness proportionate the superiority of an individual.

After two parents $\left(p_{1}\right.$ and $\left.p_{2}\right)$ are selected, they reproduce two offspring $\left(q_{c}^{1}\right.$ and $\left.q_{c}^{2}\right)$ with a single-point crossover operator (Crossover() in Figure 9). Each offspring is mutated with a mutation operator (Mutation() in Figure 9) that randomly alters the time slot assignment for each neuronal signal at the mutation rate $P_{m}$. Crossover() and Mutation() make sure that offspring never violate constraints except the delay constraint $C_{D}$.

Once $\lambda$ offspring are reproduced through parent selection, crossover and mutation, the proposed EMOA ranks $\mu+\lambda$ (i.e., $\left|P^{t} \cup Q^{t}\right|$ ) individuals and selects the top $\mu$ of them as the individuals used in the next generation $\left(P^{t+1}\right)$ with a diversity-aware selection operator (DiversityAwareSelection() in Figure 9). This operator ranks individuals based on their diversity in the objective space as well as their fitness values. It computes each individual's diversity with the notion of crowding distance [5]. A crowding distance indicates how an individual is distant from its nearest neighbors in the objective space. Thus, an individual with a higher crowding distance exists in a less crowded region in the objective space. The proposed diversity-aware selection operator plots individuals in a two dimensional space whose axes represent their fitness and diversity. Then, it determines the dominance relationships among individuals with respect to the two axes and ranks them from the ones with higher fitness and diversity to the ones with lower fitness and diversity. Finally, it selects the top $\mu$ individuals as the next generation's individuals. The proposed selection operator is designed to maintain the diversity of individuals in order to reveal the trade-offs among conflicting objectives.

At the end of each generation $(t)$, the proposed EMOA adjusts the number of offspring reproduced in the next generation $\left(\lambda_{t+1}\right)$ (OffspringSizeAdjustment () in Figure 9). $\lambda_{t+1}$ is re-computed on a generation-by-generation basis in order to adjust the density of individuals in the objective space as well as the selection pressure of individuals. In this paper, selection pressure $(\psi)$ is measured as follows:

$$
\psi=\frac{\mu+\lambda}{\mu}
$$

$\mu$ denotes the population size. Selection pressure indicates how hard individuals can survive to the next generation; a higher selection pressure means that individuals have 
lower chances to survive to the next generation. It is known that a low selection pressure significantly degrades optimization/convergence speed [8]. The proposed offspring size adjustment operator is designed to maintain a reasonably high selection pressure by adjusting $\lambda$ in Equation 6 .

The density of individuals in the objective space $(\eta)$ is measured as follows:

$$
\eta=\frac{\mu+\lambda}{\gamma}
$$

$\gamma$ denotes the total volume of the objective space. In a higher-dimensional objective space, it is harder to determine dominance relationships among individuals because individuals have higher chances to be non-dominated with each other [12]. This often leads to premature convergence, which fails to improve the quality of individuals. The proposed offspring size adjustment operator is designed to alleviate this problem by increasing $\lambda$ in Equation 7 and in turn maintaining the density of individuals in the objective space.

The size of offspring is adjusted as follows.

$$
\lambda_{t+1}=\lambda_{t}+\left(\frac{\lambda_{t-1}^{\prime}}{\lambda_{t-1}}-\frac{\lambda_{t}^{\prime}}{\lambda_{t}}\right) \lambda_{t}
$$

$\lambda_{t}^{\prime}$ denotes the number of offspring that survive to the next generation through the selection process in DiversityAwareSelection() (Figure 9). Thus, $\frac{\lambda_{t}^{\prime}}{\lambda_{t}}$ indicates the survival ratio of offspring. If is is lower than the survival ratio at the previous generation $\left(\frac{\lambda_{t-1}^{\prime}}{\lambda_{t-1}}\right)$, the proposed operator considers that convergence/evolution does not proceed well due to a lack of enough selection pressure and/or individual density in the objective space. Therefore, the operator increases the number of offspring reproduced in the next generation $\left(\lambda_{t+1}\right)$. Conversely, if $\frac{\lambda_{t}^{\prime}}{\lambda_{t}}>\frac{\lambda_{t-1}^{\prime}}{\lambda_{t-1}}$, the operator decreases $\lambda_{t+1}$.

\section{EVALUATION}

This section evaluates the proposed EMOA in Neuronal TDMA through simulations.

\subsection{Simulation Configurations}

This paper simulates a neuronal network that contains 43 neurons (Figures 10). 11 sensors are evenly distributed in the network. Although a number of studies have investigated the topology shapes of neuronal networks, Diffusion Limited Aggregation (DLA) is a common method to represent and generate their tree topology shapes [17]. This paper uses a similar random tree-like topology that mimics a dendritic tree among neurons [17] (Figure 10).

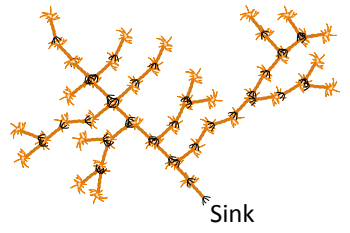

Figure 10: A Simulated Neuronal Network

The proposed EMOA is configured with a set of parameters shown in Table 1. It is compared with two well-known existing EMOAs: NSGA-II [5] and SPEA2 [27]. Each experimental result is obtained from 20 independent experiments.
Table 1: EMOA Configurations

\begin{tabular}{|l|c|}
\hline Parameter & Value \\
\hline \hline The initial population size $\left(\mu_{0}\right.$ in Figure 9$)$ & 100 \\
The initial offspring size $\lambda_{0}\left(\mu_{0}\right.$ in Figure 9$)$ & 100 \\
Mutation rate $\left(P_{m}\right.$ in Figure 9$)$ & $10 \%$ \\
The max. number of generations $\left(g_{\max }\right.$ in Figure 9$)$ & 100 \\
\hline
\end{tabular}

\subsection{Simulation Results}

Figure 11 shows how individuals increase the union of the hypervolumes that they constrained-dominate in the objective space as the number of generations grows in the proposed EMOA, NSGA-II and SPEA2. The hypervolume metric quantifies the optimality and diversity of individuals [28]. A higher hypervolume means that individuals are closer to the Pareto-optimal front and more diverse in the objective space. As Figure 11 shows, the proposed EMOA rapidly increases its hypervolume measure in the first 10 generations and converges around the 60th generation. At the last generation, all individuals are non-constrained-dominated in the population. This verifies that the proposed EMOA allows individuals to efficiently evolve and improve their quality and diversity within 100 generation.

Figure 11 also compares evolutionary convergence among the proposed EMOA, NSGA-II and SPEA2. All the three EMOAs initially increase hypervolume measures at a similar rate; however, the proposed EMOA converges to a higher hypervolume measure than NSGA-II and SPEA2. Figure 11 shows that the proposed EMOA outperforms NSGA-II and SPEA2 in the quality and diversity of individuals.

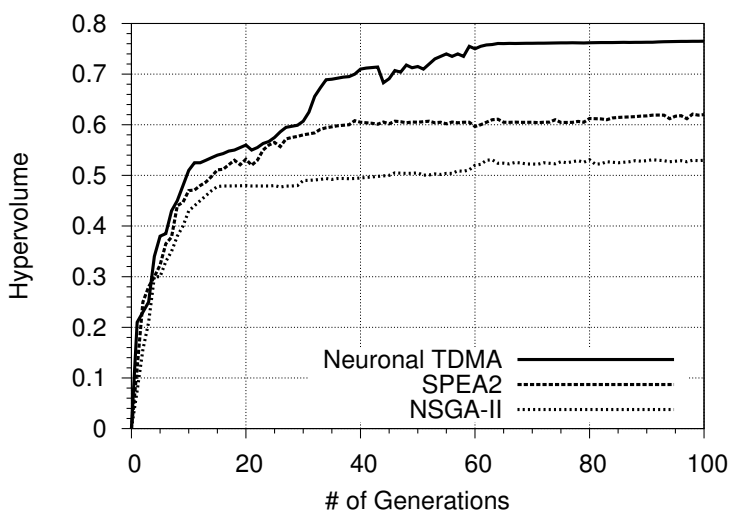

Figure 11: Hypervolume

Table 2 compares the proposed EMOA, NSGA-II and SPEA2 with the coverage metric $(\mathcal{C}$-metric). This metric compares two sets of individuals [29]. Given individual sets $A$ and $B, \mathcal{C}(A, B)$ measures the fraction of individuals in $B$ that at least one individual in $A$ dominates:

$$
\mathcal{C}(A, B)=\frac{|\{b \in B \mid \exists a \in A: a \succ b\}|}{|B|}
$$

The $\mathcal{C}$-metric values in Table 2 are computed with a set of individuals that each EMOA produces at the last generation. As shown in Table 2, $\mathcal{C}$ (Neuronal TDMA, NSGA-II) is greater than $\mathcal{C}$ (NSGA-II, Neuronal TDMA) $(0.55>0.0)$. Also, $\mathcal{C}($ Neuronal TDMA, SPEA2) $>\mathcal{C}($ SPEA2, Neuronal 
TDMA) $(0.45>0.0)$. These results mean that the proposed EMOA outperforms NSGA-II and SPEA2 in the quality of individuals. No individuals of NSGA-II and SPEA2 can dominate the individuals of the proposed EMOA.

Table 2: C-metric Comparison

\begin{tabular}{|c||c|}
\hline & $\mathcal{C}$-metric value \\
\hline $\mathcal{C}$ (Neuronal TDMA, NSGA-II) & 0.55 \\
\hline $\mathcal{C}$ (NSGAII, Neuronal TDMA) & 0.0 \\
\hline \hline $\mathcal{C}$ (Neuronal TDMA, SPEA2) & 0.45 \\
\hline $\mathcal{C}$ (SPEA2, Neuronal TDMA) & 0.0 \\
\hline
\end{tabular}

Figure 12 illustrates the diversity of individuals with the distribution metric This metric measures the degree of uniform distribution of individuals in the objective space. It is computed as the standard deviation of Euclidean distances among individuals:

$$
\sqrt{\frac{\sum_{i=1}^{N-1}\left(d_{i}-\bar{d}\right)^{2}}{N-1}}
$$

$d_{i}$ denotes the Euclidean distance between a given individual (the $i$-th individual in the population) and its closest neighbor in the objective space. $\bar{d}$ denotes the mean of $d_{i} . N$ denotes the number of individuals in the population. The objective space is normalized to compute the distribution metric. Lower distribution means that individuals are more uniformly (or evenly) distributed.

As shown in Figure 12, all three EMOAs improve the diversity of individuals as the number of generations grows. At the last generation, the proposed EMOA's distribution is less than the half of NSGAII's. Along with the evaluation with the hypervolume metric, the proposed EMOA outperforms NSGA-II and SPEA2 in the diversity of individuals.

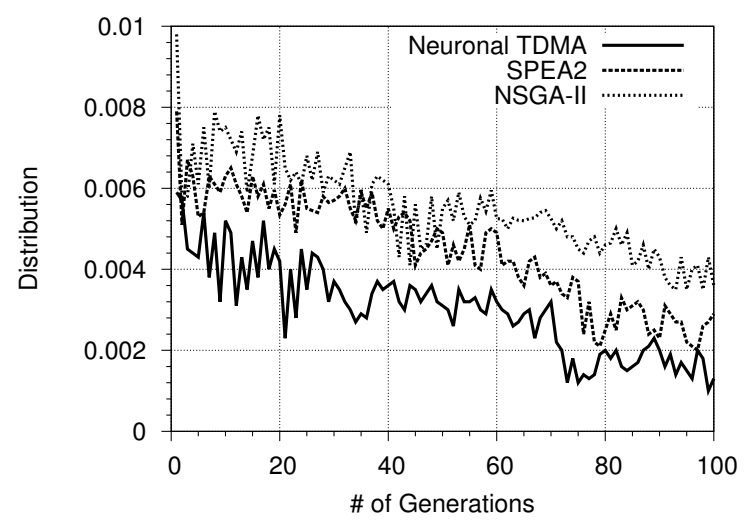

Figure 12: Distribution

Table 3 shows the average of each objective value. A value in parentheses indicates a standard deviation of objective values that an EMOA yields in 20 independent simulations. As this table illustrates, Neuronal TDMA yields the best objective values on average in all three objectives.

Table 4 shows how Neuronal TDMA yields three objective values, respectively, subject to different signaling delay constraints $\left(C_{D}\right)$. Neuronal TDMA successfully meets both USD constraints. Given a more strict USD constraint
Table 3: Average Objective Values

\begin{tabular}{|c||c|c|c|}
\hline & $f_{Y}$ & $f_{F}$ & $f_{D}$ \\
\hline \hline Neuronal TDMA & $22(4.33)$ & $0.10(0.33)$ & $22.86(5.11)$ \\
\hline NSGA-II & $16.56(3.09)$ & $0.07(0.99)$ & $31.87(7.33)$ \\
\hline SPEA2 & $18.34(3.96)$ & $0.08(0.10)$ & $25.99(6.44)$ \\
\hline
\end{tabular}

$\left(C_{D}=17\right)$, it improves its $f_{D}$ while degrading $f_{Y}$ and $f_{F}$. Table 4 demonstrate that Neuronal TDMA can provide different optimization results under different constraints. This means that it allows IBSAN designers to examine various "what-if" analyses. For example, they can examine whether they can sacrifice $f_{Y}$ by $35 \%$ and $f_{F}$ by $50 \%$ to improve $f_{D}$ by $25 \%$. This way, Neuronal TDMA aids IBSAN designers to make well-informed scheduling decisions for signaling in IBSANs.

Table 4: Objective Values with Constraints

\begin{tabular}{|c||c|c|c|}
\hline & $f_{Y}$ & $f_{F}$ & $f_{D}$ \\
\hline \hline$C_{D}=21$ & $18(3.1)$ & $6.1(0.9)$ & $20.17(0.89)$ \\
\hline$C_{D}=17$ & $14(2.5)$ & $3.4(0.4)$ & $16.78(1.01)$ \\
\hline
\end{tabular}

\section{RELATED WORK}

Due to the numerous problems (e.g. disability of limbs requiring artificial limbs) and diseases (e.g. Alzheimer disease), numerous research work have been conducted in the field of neuroscience. These studies ranged from understanding the signaling of the neuronal networks in specific parts (clustered areas), to more recently understanding the signaling of individual neurons and their role in the networks. As described earlier, numerous works have gone into preparing environmental substrates $[21,22,10]$ that can allow neurons to be grown, in particular grown in a long period of time, in order for efficient study to be performed. The challenge for this is due to the fact that neuron networks that are artificially grown, will usually result in neuron death. Majority of work in the last decade has focused on neuron networks grown in two dimensions. Recently, new approaches have been developed to allow neurons to be grown in three dimensions, which leads to a more realistic topology of real neuronal networks. All these various solutions, are all applicable towards our scheduling protocol that we have proposed in this paper. Numerous research works have also gone into understanding the characteristics of the neuron networks, and their robustness. For example Kotsavasiloglu et al [14, 15] developed computational models to understand the robustness of neuronal networks with healthy neurons, and how their performance reacts when synapse failures or changes in refractory periods or excitation synapse ratio occur. The authors were able to show the tremendous robustness exhibited by the neuronal networks, in particular for cases of Alzheimer's and Parkinsons. Graph Theory has also been investigated in neuronal networks [3]. The study discovered that the neuronal network connectivity is governed by a Gaussian distribution, and if the network connectivity increases, this could lead to a percolation transition occurring at critical synaptic strength. Signalling neurons have also been investigated for artificially grown neuronal networks, and one example is by [9], where LED matrices were used to invoke neuron signaling. 


\section{CONCLUSIONS}

The proposed framework, Neuronal Network, is designed to optimize the signaling schedule for nanomachines in IBSANs. Simulation results verify this and demonstrates that Neuronal Network outperforms conventional EMOAs.

\section{ACKNOWLEDGMENT}

The authors thank Paskorn Champrasert for his contribution. This work is supported in part by Science Foundation Ireland via the "A biologically inspired framework supporting network management for the future Internet" starting investigator award (grant no. 09/SIRG/I1643).

\section{REFERENCES}

[1] B. Atakan, S. Balasubramaniam, and O. B. Akan. Body area nanonetworks with molecular communications in nanomedicine. IEEE Communications Magazine, January 2012.

[2] S. Balasubramaniam, N. T. Boyle, A. Della-Chiesa, F. Walsh, A. Mardinoglu, D. Botvich, and A. Prina-Mello. Development of artificial neuronal networks for molecular communication. Nano Communication Networks, 2(2-3), 2011.

[3] I. Breskin, J. Soriano, E. Moses, and T. Tlusty. Percolation in living neural networks. Physical Review Letters, 97, 2006.

[4] R. Cajal. Histology of nervous system of man and vertebrates. Oxford University Press, 1995.

[5] K. Deb, S. Agrawal, A. Pratab, and T. Meyarivan. A fast elitist non-dominated sorting genetic algorithm for multi-objective optimization: NSGA-II. In Proc. Conf. Parallel Problem Solving from Nature, 2000.

[6] T. Gabay, E. Jakobs, E. Ben-Jacob, and Y. Hanein. Engineered self-organization of neural networks using carbon nanotube clusters. Physica A, 350(2-4), 2005.

[7] L. P. Gine and I. F. Akyildiz. Molecular communication options for long range nanonetworks. Computer Networks, 53, 2009.

[8] D. E. Goldberg. Genetic Algorithms in Search, Optimization and Machine Learning. Addison, 1989.

[9] N. Grossman, K. Nikolic, and P. Degenaar. The neurophotonic interface: stimulating neurons with light. The Neuromorphic Engineer, 2008.

[10] S. Hiyama and Y. Moritani. Molecular communication: Harnessing biochemical materials to engineer biomimetic communication systems. Nano Communication Network, 1(1), 2010.

[11] J. B. Hursh. Conduction velocity and diameter of nerve fibers. Amer. J. Physiol., 127, 1939.

[12] H. Ishibuchi, N. Tsukamoto, Y. Hitotsuyanagi, and Y. Nojima. Effectiveness of Scalability Improvement Attempts on the Performance of NSGA-II for Many-Objective Problems. In Proc. ACM Conference on Genetic and Evol. Computat., 2008.

[13] S. B. Jun, M. R. Hynd, N. Dowell-Mesfin, K. L. Smith, J. N. Turner, W. Shain, and S. J. Kima. Low-density neuronal networks cultured using patterned polyl-L-lysine on microelectrode arrays. Journal of Neurosci. Methods, 160(2), 2007.

[14] A. Kalampokis, C. Kotsavasiloglou, P. Argyrakis, and S. Baloyannis. Robustness of biological neural networks. Physica A, 317, 2003.

[15] C. Kotsavasiloglou, A. Kalampokis, P. Argyakis, and S. Baloyannis. Modeling signal transmission and robustness in biological neural networks. Int'l $J$. Biology and Biomedical Engineering, 1(2), 2007.

[16] P. Lio and S. Balasubramaniam. Opportunistic routing through conjugation in bacteria communication nanonetwork. Nano Comm. Networks, 3(1), 2012.

[17] A. Luczak. Measuring neuronal branching patterns using model-based approach. Front. Comput. Neurosci., 4(135), 2010.

[18] E. N. McNamee, K. M. Ryan, D. Kilroy, and T. J. Connor. Noradrenalin induces IL-1ra and IL-1 type II receptor expression in primary glial cells and protects against il-1b-induced neurotoxicity.

[19] M. Moore, A. Enomoto, T. Nakano, R. Egashira, T. Suda, A. Kayasuga, H. Kojima, H. Sakakibara, and K. Oiwa. A design of a molecular communication system for nanomachines using molecular motors. In Proc. IEEE Int'l Conference on Pervasive Computing and Communications Workshops, 2006.

[20] F. Morin, N. Nishimura, L. Griscomb, B. LePioufle, H. Fujita, Y. Takamura, and E. Tamiya. Constraining the connectivity of neuronal networks cultured on microelectrode arrays with microSSuidic techniques: A step towards neuron-based functional chips. Biosensors and Bioelectronics, 21, 2006.

[21] T. Nakano. Biologically-inspired network systems: A review and future prospects. IEEE Trans, Systems, Man and Cybernetics: Part C, 41(4), 2011.

[22] T. Nakano, J. W. Shuai, T. Koujin, T. Suda, Y. Hiraoka, and T. Haraguchi. Biological excitable media with non-excitable cells and calcium signaling. Nano Communication Networks, 1(1), 2010.

[23] T. Nakano, T. Suda, M. Moore, R. Egashira, A. Enomoto, and K. Arima. Molecular communication for nanomachines using intercellular calcium signaling. In Proc. IEEE Int'l Conf. on Nanotechnology, 2005.

[24] G. Stuart, J. Schiller, and B. Sakmann. Action potential initiation and propagation in rat neocortical pyramidal neurons. Journal of Physiology, 505.3, 1997.

[25] T. Suda, M. Moore, T. Nakano, R. Egashira, and A. Enomoto. Exploratory research on molecular communication between nanomachines. In Proc. ACM Genetic and Evol. Computat. Conference, 2005.

[26] C. Wyart, C. Ybert, L. Bourdieu, C. Herr, C. Prinz, and D. Chatenay. Constrained synaptic connectivity in functional mammalian neuronal networks grown on patterned surfaces. J. Neurosci. Methods, 117(2), 2002.

[27] E. Zitzler, M. Laumanns, and L. Thiele. SPEA2: Improving the strength pareto evolutionary algorithm for multiobjec- tive optimization. In Evol. Methods for Design, Optimisation and Control with Application to Industrial Problems. 2002.

[28] E. Zitzler and L. Thiele. Multiobjective optimization using evolutionary algorithms: A comparative study. In Proc. Int'l Conf. on Parallel Problem Solving from Nature, 1998.

[29] E. Zitzler and L. Thiele. Multiobjective evolutionary algorithms: a comparative case study and the strength pareto approach. IEEE Trans. Evol. Computat., 3(4), 1999. 